\title{
Transplacental passage of vancomycin in the ex vivo human perfusion model
}

Presented at the Annual Scientific Meeting and Symposium of the Infectious Diseases Society for Obstetrics and Gynecology, Hyannis, Massachusetts, MA, August 7-9, 2003

\author{
Michael D. Hnat, Julie Gainer, Roger E. Bawdon and George D. Wendel Jr \\ Division of Maternal-Fetal Medicine, Department of Obstetrics and Gynecology, University of Texas, \\ Dallas, TX
}

Objectives: To determine maternal-fetal transplacental passage of vancomycin in the ex vivo human placental perfusion model.

Methods: Six term placentas were collected immediately after delivery and perfused with physiologic medium using the single cotyledon perfusion system. Vancomycin was added to the maternal medium and perfused through the maternal circulation of the cotyledon. Over a I-h period in an open system, samples of the perfusate were collected at defined intervals from the fetal venous catheter and from the maternal effluence to assess vancomycin transfer. Thereafter, the system was closed for $\mathrm{I}-5 \mathrm{~h}$ to establish accumulation. Transport fraction and clearance indexes were calculated by perfusing antipyrine ${ }^{14} \mathrm{C}$ (positive control). Vancomycin was estimated by high pressure liquid chromatography and antipyrine ${ }^{14} \mathrm{C}$ concentration was determined by liquid scintillation.

Results: Mean vancomycin maternal peak and trough concentrations ranged from 30.0 to $51.5 \mu \mathrm{g} / \mathrm{ml}$ and 7.7 to $16.4 \mu \mathrm{g} / \mathrm{ml}$, respectively. Clearance indexes were minimal with a mean peak range of $0.000-0.080$ and a mean trough range of $0.00-0.17$. For each placenta, transport fraction for antipyrine ${ }^{14} \mathrm{C}$ was $>1.85$ with a single pass of $>40 \%$. No accumulation of vancomycin was noted when the system was closed for I-5 h. The mean peak clearance index was zero after perfusing the placenta for up to $5 \mathrm{~h}$ with $35.8 \mu \mathrm{g} / \mathrm{ml}$ of vancomycin.

Conclusion: Transplacental passage of vancomycin was minimal in the ex vivo human placental perfusion model, with no detectable accumulation.

Key words: Vancomycin; Placenta; Fetus; Beta Streptococcus; Pregnancy

In 2002, the Centers for Disease Control released new guidelines for the prevention of group B streptococcal (GBS) disease in the neonate ${ }^{1}$. The American College of Obstetricians and Gynecologists had concurred with their recommendations which include universal prenatal culture-based screening at 35-37 weeks together with updated chemoprophylaxis regimens for women with penicillin allergies ${ }^{2}$. Penicillin remains the firstline agent for intrapartum prophylaxis. Cefazolin is recommended for women with a reported penicillin allergy at low risk for complications ${ }^{1-4}$.

Correspondence to: Michael D. Hnat, UT Southwestern Medical Center, 5323 Harry Hines Blvd, Dallas, TX 75390-9032, USA.

Email: Michael.Hnat@UTSouthwestern.edu

(C) 2004 Parthenon Publishing. A member of the Taylor \& Francis Group

DOI: I0. I080/I064744040000982 I 
Clindamycin or erythromycin is indicated for those who are penicillin-allergic and are at high risk for anaphylaxis or at high risk for anaphylaxis complications secondary to certain medical conditions such as asthma ${ }^{1}$.

Resistance of GBS isolates to erythromycin and clindamycin has increased over the past few years ${ }^{5-}$ ${ }^{8}$. Therefore, susceptibility testing to these antibiotics is recommended in the new GBS prophylaxis guidelines. Vancomycin is now advised for isolates that show in vitro resistance to clindamycin or erythromycin or in cases where the susceptibility of the prenatal isolate is unknown ${ }^{1}$.

Data are lacking on the relative effectiveness of vancomycin for the prevention of GBS in the neonate. In addition, information regarding vancomycin pharmacokinetics in pregnancy is limited. Two studies have observed transplacental crossing of vancomycin in women with amnionitis when administered over several days ${ }^{9,10}$. Bourget and colleagues investigated transplacental transfer of vancomycin in a gravid woman with amnionitis at 26 weeks ${ }^{9}$. Reyes and others found adequate vancomycin levels in 8 of 10 pregnant women with amnionitis in the second and third trimester ${ }^{10}$. It is unknown whether vancomycin crosses from the maternal to the fetal circulation in a term placenta without infection. Therefore, our objective was to quantify the maternal to fetal transplacental passage of vancomycin in the noninfected ex vivo human placental perfusion model.

\section{MATERIALS AND METHODS}

Term placentas were collected from either Cesarean sections or vaginal deliveries in accordance with the University of Texas Southwestern Medical Center Institutional Review Board for Human Studies guidelines, and transported to the laboratory in normal saline immediately after delivery.

The single cotyledon placental perfusion system was used as described by Chalier ${ }^{11}$. A fetal artery and vein on the chorionic plate were cannulated with $3.0 \mathrm{~F}$ and $5.0 \mathrm{~F}$ catheters, respectively. The fetal circulation of the selected cotyledon was gently perfused with Eagle's minimal essential media plus 3\% bovine albumin and 0.5 to 1.0 units $/ \mathrm{ml}$ of heparin (Sigma
Chemical Co, St Louis, MO). The cotyledon was initially examined for vascular integrity.

The localized cotyledon and surrounding placental tissue were transferred to a temperature-controlled chamber $\left(37^{\circ} \mathrm{C}\right)$ where the fetal circulation was perfused in a closed system at $4.5-$ $5.0 \mathrm{ml} / \mathrm{min}$ for $20 \mathrm{~min}$ to remove any residual blood and to stabilize perfusion pressure at 35$50 \mathrm{mmHg}$. Those placental cotyledons that failed to reach a stable baseline pressure or were found to have a vascular leak were discarded. Lastly, two 18gauge needles were inserted into the intervillous space of the cotyledon to re-establish the maternal circulation. Maternal flow rate was $17 \mathrm{ml} / \mathrm{min}$.

The maternal and fetal compartments consisted of $150 \mathrm{ml}$ Eagle's minimal essential medium (pH 7.2-7.4), aerated with 95\% oxygen and 5\% carbon dioxide and continually mixed by magnetic stirring bar. Transfer studies were performed with vancomycin hydrochloride (Sigma). Vancomycin was added to the maternal medium in concentrations similar to maternal serum peak and trough levels. In addition, experiments were first conducted in an open-open system to determine transport fraction, then in a closed-closed recirculation system to determine accumulation. The clearance index and accumulation of vancomycin were also determined. Fluid aliquots from both maternal and fetal compartments were collected for analysis.

A total of 6 placentas met criteria for inclusion in the study. A trough transfer study for vancomycin was performed in 1 placenta and a peak study in 1 other. Studies were completed first in an open system for $2 \mathrm{~h}$ and then in a closed system. Both peak and trough transfer studies were conducted in the other 4; trough studies were performed in an open system and then in a closed system, followed by peak studies in an open system and then in a closed system.

Lastly, transport fraction and clearance indexes were determined for each placenta by means of the ${ }^{14} \mathrm{C}$ antipyrine reference method of Chalier ${ }^{5}$. Placentas were studied in a non-recirculating (open) system for $60 \mathrm{~min}$ to determine the transport fraction of ${ }^{14} \mathrm{C}$ antipyrine. Greater than $40 \%$ maternal-fetal transfer of antipyrine was considered an indication of a successful maternalfetal match. 
Vancomycin and antipyrine ${ }^{14} \mathrm{C}$ concentrations were measured by high pressure liquid chromatography (HPLC) and liquid scintillation, respectively. Reproducibility studies on the vancomycin assay were performed. The HPLC analysis of vancomycin was carried out on Waters Associate instruments (Medford, MA). For HPLC analysis, the perfusion sample was extracted with $0.5 \mathrm{ml}$ of $10 \%$ trichloroacetic acid. The samples and standards were mixed and centrifuged at 2000 $\times \mathrm{g}$ for $10 \mathrm{~min}$. The clear extract was injected into a Phenomenex $\mathrm{C}_{18} \mu$ Bond-a-pak column (Torrance, CA). For HPLC, $\mathrm{pH}$ was 6.1 at a sensitivity of $.005 \mathrm{AU}$ and a detector wavelength of 2902. The buffer flow rate was $2.0 \mathrm{ml} / \mathrm{min}$ and an injection volume of $80 \mu \mathrm{l}$ was used. The retention time was about $6.0 \mathrm{~min}$. All concentrations of vancomycin were determined by measuring the peak height of the standards and specimen. The HPLC method was validated; the reproducibility data are not shown.

\section{RESULTS}

For the HPLC determination of vancomycin concentrations, reproducibility studies provided values with a minimum sensitivity of $0.1 \mu \mathrm{g} / \mathrm{ml}$, and within-batch and between-batch reproducibility of $>90 \%$. Transport fraction and clearance indexes of vancomycin were determined at two concentrations, peak and trough. For the maternal effluence, peak and trough levels are shown in Table 1. The mean trough level was $11.7 \mu \mathrm{g} / \mathrm{ml}$ with a range of $7.7-16.4 \mu \mathrm{g} / \mathrm{ml}$, and the mean peak level was $37.2 \mu \mathrm{g} / \mathrm{ml}$ with a range of 30.0 $51.5 \mu \mathrm{g} / \mathrm{ml}$. At trough levels, the concentration of vancomycin in the fetal circulation was below the minimum sensitivity of the assay $(<0.1 \mu \mathrm{g} /$

Table I Mean vancomycin concentrations $(\mu \mathrm{g} / \mathrm{ml})$, troughs and peaks, in placentas I-6

\begin{tabular}{lcc}
\hline Placenta & Trough & Peak \\
\hline 1 & - & 51.5 \\
2 & 16.4 & 33.0 \\
3 & 11.7 & 33.0 \\
4 & 7.7 & 40.1 \\
5 & 14.8 & - \\
6 & 8.0 & 30.0 \\
\hline
\end{tabular}

$\mathrm{ml}$ ) in 3 of the 5 trough levels and in 2 of the 5 peak levels. Thus, the clearance index could only be calculated in the studies where vancomycin concentrations were detected in the fetal circulation. When the concentration of vancomycin was not detected in the fetal concentration, the clearance index was considered zero. For both peak and trough concentrations, the clearance indexes were minimal (Table 2).

The transport factor for antipyrine ${ }^{14} \mathrm{C}$ was $>1.85$ with a single pass transfer greater than $40 \%$ for each placenta. When the system was closed for 1 to $5 \mathrm{~h}$, no fetal accumulation was noted for peak and trough concentrations of vancomycin.

\section{DISCUSSION}

Our data suggest that the clearance index of vancomycin is low at both peak and trough concentrations in the ex vivo placental perfusion model. However, preliminary data from a present study in our lab suggest a higher clearance index of vancomycin in vivo. Certain aspects of the model may have biased the results: for example, vancomycin precipitates when mixed with more than 1 unit/ml heparin ${ }^{12,13}$. Although the media used in this experiment contained less than or equal to $1.0 \mathrm{unit} / \mathrm{ml}$ of heparin, a vancomycinheparin complex may have been formed, decreasing vancomycin's transplacental passage. HPLC revealed appropriate and constant levels of vancomycin in the maternal effluence containing 0.5-1.0 unit/ml of heparin. Review of the HPLC data revealed artifact present for placenta 3 , falsely elevating the clearance index to 0.08 . Of note, the presence of antipyrine ${ }^{14} \mathrm{C}$ also interfered with the HPLC and falsely elevated the concentration of

Table 2 Mean vancomycin clearance indexes, troughs and peaks, in placentas I-6

\begin{tabular}{lll}
\hline Placenta & Trough & Peak \\
\hline 1 & - & 0.035 \\
2 & 0.00 & 0.000 \\
3 & 0.17 & 0.080 \\
4 & 0.05 & 0.014 \\
5 & 0.00 & - \\
6 & 0.00 & 0.000 \\
\hline
\end{tabular}


vancomycin. Thus, antipyrine ${ }^{14} \mathrm{C}$ was infused at the end of the experiment after all the samples for vancomycin were collected in the fetal and maternal compartments.

Most drugs cross the placenta by simple diffusion. Transplacental diffusion of substances is dependent upon many factors, such as molecular size or weight, ionic charge, lipid solubility and the circulating concentration not bound to plasma proteins ${ }^{14}$. Approximately a third to a half of vancomycin is bound to plasma proteins and is unavailable for transfer ${ }^{15}$. Furthermore, hydrophilic substances cross more slowly than lipophilic substances and the placental extracellular pores have limited surface area. In addition, transfer of any substance or drug through the pores is dependent upon its molecular weight. Substances with a molecular weight of less than 500 daltons will usually cross the placenta ${ }^{14}$ and rate of transfer is proportional to molecular weight (i.e. a large complex molecule will diffuse at a slower rate than a smaller molecule). Vancomycin is a complex tricyclic glycopeptide with a molecular weight of approximately 1500 daltons ${ }^{15}$. In our ex vivo model, the transfer rate or clearance index for vancomycin was almost zero for peak and trough concentrations.

The pharmacokinetics of vancomycin may be altered in vivo by the physiologic changes of pregnancy. In non-pregnant subjects, $30-55 \%$ of vancomycin is bound to plasma proteins and 90\% is excreted by glomerular filtration ${ }^{15,16}$. Plasma proteins, glomerular filtration rate and plasma volume are increased during pregnancy, decreasing the total unbound concentration of the drug available to cross the placenta. This may further decrease the concentration available for placental transfer. In non-pregnant subjects, plasma levels reach a concentration of $15-30 \mu \mathrm{g} / \mathrm{ml}$ approximately $1 \mathrm{~h}$ after intravenous administration of $1 \mathrm{~g}$ vancomycin over $1-2 \mathrm{~h}$.

Previous studies have shown that vancomycin crosses membrane barriers only when infection and inflammation are present ${ }^{9,10,16}$. With meningitis, cerebral spinal fluid (CSF) levels of vancomycin range from $7 \%$ to $30 \%$ of the serum concentration ${ }^{16}$. Without meningitis, CSF levels are undetectable ${ }^{16}$. In two studies, vancomycin concentration reached therapeutic levels in the fetal circulation when treating the mother for overt amnionitis in a preterm gestation in hopes of prolonging the pregnancy ${ }^{9,10}$ and adequate levels were obtained after multiple doses. The administration of 1 or 2 doses of vancomycin may be inadequate to obtain therapeutic levels in the fetal compartment in the presence or absence of inflammation.

In conclusion, our results show that there is minimal or no transport of vancomycin into the fetal compartment in the ex vivo placental profusion model. In addition, vancomycin concentration and pharmacokinetics may be altered by the normal physiologic changes of pregnancy affecting placental passage. Therefore, additional studies using vancomycin in pregnancy should be undertaken to assess its effectiveness in GBS chemoprophylaxis.

\section{ACKNOWLEDGMENTS}

We would like to thank those who helped obtain the placentas for this study, especially the Parkland Memorial Hospital midwives, residents, nurses and clerks.

\section{REFERENCES}

1. Centers for Disease Control. Prevention of perinatal group B streptococcal disease. MMWR 2002;51:1-23

2. American College of Obstetricians and Gynecology. Prevention of early-onset group B streptococcal disease in newborns. ACOG Committee Opinion 279, December 2002
3. Kelkar PS, Li JT. Cephalosporin allergy. N Engl J Med 2001;345:804-9

4. Centers for Disease Control. Sexually transmitted diseases treatment guidelines. MMWR 2002;51: 28-9 
5. Andrews JJ, Diekema DJ, Hunter SK, et al. Group B streptococci causing neonatal bloodstream infection: asusceptibility and serotyping results from SENTRY center in the Western Hemisphere. Am J Obstet Gynecol 2000;183:859-62

6. Fernandez M, Hickman ME, Baker CJ. Antimicrobial susceptibilities of group B streptococci isolated between 1992 and 1996 from patients with bacteremia or meningitis. Antimicrob Agents Chemother 1998;42:1517-9

7. Lin FYC, Azimi PH, Wiseman LE, et al. Antibiotic susceptibility profiles for group B streptococci isolated from neonates, 1995-1998. Clin Infect Dis 2000;31:76-9

8. Bland ML, Vermillion ST, Soper DE, Austin M. Antibiotic resistance patterns of group B streptococci in late third-trimester rectovaginal cultures. Am J Obstet Gynecol 2001;184:1124-6

9. Bourget P, Fernandez H, Delouis C, Ribou F. Transplacental passage of vancomycin during the second trimester of pregnancy. Obstet Gynecol 1991;78:908-11

10. Reyes MP, Ostrea EM, Cabinian AE, Schmitt C, Rintelmann W. Vancomycin during pregnancy: does it cause hearing loss or nephrotoxicity in the infant? Am J Obstet Gynecol 1989;161:977-81

RECEIVED 10-02-03; ACCEPTED 01-16-04
11. Chalier JC. Criteria for evaluating perfusion experiments and presentation results. Contrib Gynecol Obstet 1985;13:32-9

12. Kershaw BP, Monnier HL, Mason JH. Visual compatibility of premixed theophylline or heparin with selected drugs for iv administration. Am J Hosp Pharm 1993;50:1360-3

13. Najari A, Rusho WJ. Compatibility of commonly used bone marrow transplant drugs during Y-site delivery. Am J Health Syst Pharm 1997;54:181-4

14. Cunningham FG, et al., eds. Williams Obstetrics. New York: McGraw-Hill, 2001:138

15. Fekety R. Vancomycin, teicoplanin, and the streptogramins: quinupristin and dalfopristin. In: Mandell GL, Bennett JE, Dolin R, eds. Principles and Practices of Infectious Disease. Philadelphia: Churchill Livingstone, 2000:382-92

16. McEvoy GK. American Hospital Formulary Service Drug Information. Bethesda, MD: American Society of Health-System Pharmacists, 2003: $470-4$ 


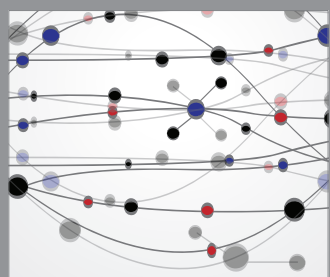

The Scientific World Journal
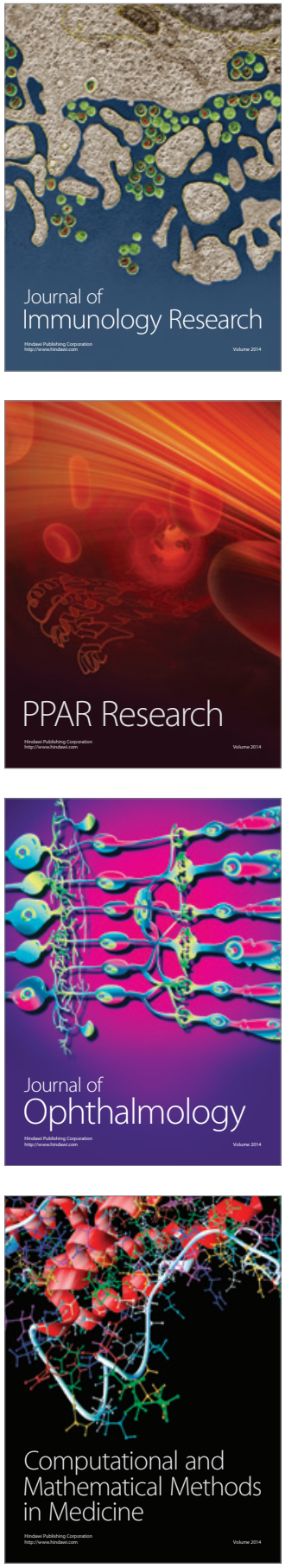

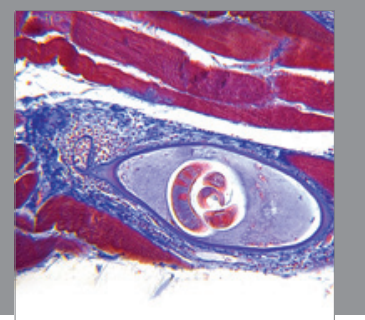

Gastroenterology

Research and Practice
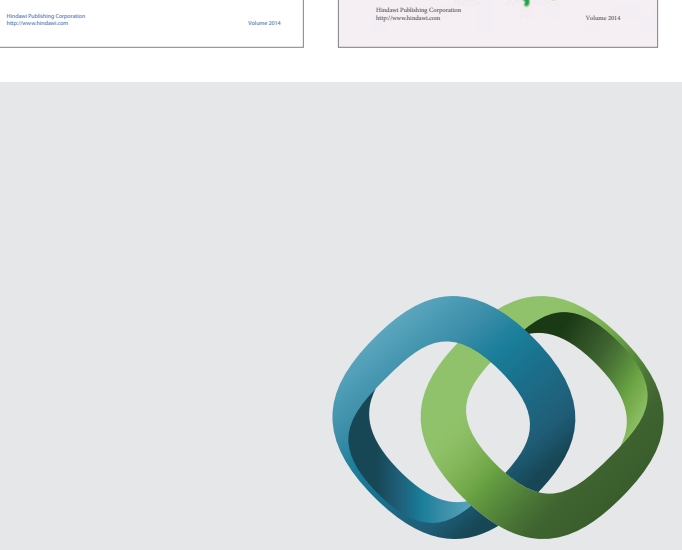

\section{Hindawi}

Submit your manuscripts at

http://www.hindawi.com
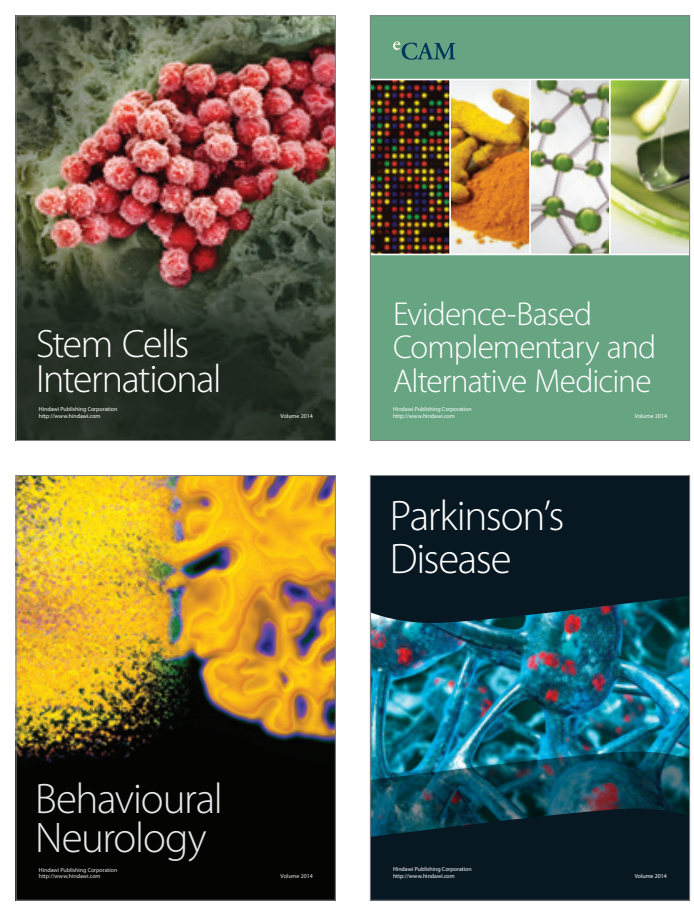

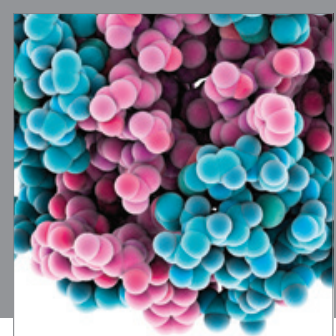

Journal of
Diabetes Research

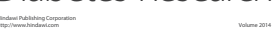

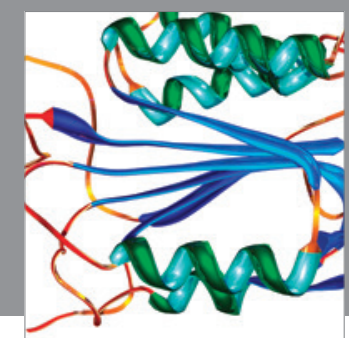

Disease Markers
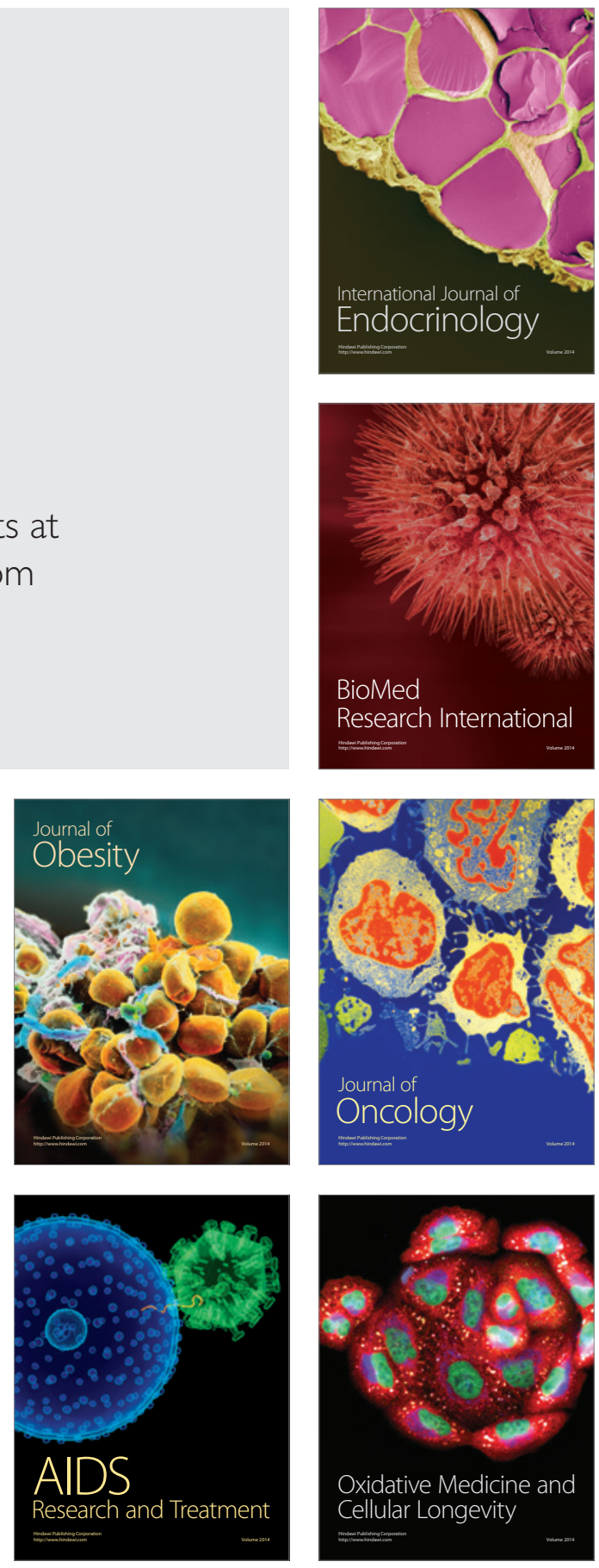